\title{
Fundamentals of the Application of Matrix- Assisted Laser Desorption-Ionization Mass Spectrometry to Low Mass Poly(methylmethacrylate) Polymers
}

\author{
Barbara S. Larsen, William J. Simonsick, Jr., and Charles N. McEwen \\ E. I. du Pont de Nemours \& Company, Wilmington, Delaware, USA
}

\begin{abstract}
Matrix-assisted laser desorption-ionization (MALDI) time of flight is shown to give a molar peak area response for isolated methylmethacrylate oligomers that have 25 and 50 repeat units when run on three different instruments in reflectron or linear mode and using three different matrix materials. In addition, fragmentation was not observed in any of the three different matrices or at higher laser power. No spectral differences were observed for syndiotactic and isotactic methylmethacrylate oligomers. These results suggest that the low most probable peak values observed for narrow distribution poly(methylmethacrylate) standards by MALDI mass spectrometry are not the result of mass discrimination or fragmentation. (J Am Soc Mass Spectrom 1996, 7, 287-292)
\end{abstract}

$\mathrm{M}$ ass spectrometry is a viable technique for analysis of synthetic polymers and has been used extensively for the analysis of additives, impurities, and pyrolysis products [1-3]. Recent advances in ionization methods have made it possible to characterize intact polymers by mass spectrometry. These methods include field desorption (FD) [4, 5], fast-atom bombardment (FAB) [6], secondary-ion mass spectrometry (SIMS) [7, 8], laser desorption (LD) [9-11], potassium ionization of desorbed species (KIDS) [12, 13], electrospray ionization (ESI) $[14,15]$, and matrixassisted laser desorption-ionization (MALDI) [16]. In particular, the introduction of MALDI and ESI ionization techniques has dramatically increased the mass range for molecular weight analyses by mass spectrometry. MALDI recently was shown to be applicable to ionization of starburst dendrimers that have molecular weights above 1,000,000 (P. Savickas, private communication). Electrospray has produced ions from polyethylene glycols that have molecular weights to $5,000,000[14]$.

Mass spectrometry has the potential to characterize polymers and oligomers with greater accuracy and precision for molecular weight and end group determination than methods such as size exclusion chromatography (SEC), laser light scattering, vapor phase osmometry, or nuclear magnetic resonance spectroscopy. Polymer characteristics that are potentially available

Address reprint requests to Dr. Barbara Larsen, The DuPont Company, P.O. Box 80228, Wilmington, DE 19880-0228. through mass spectrometric analysis are repeat unit mass, end group mass, cyclic structure determination, number average $\left(M_{n}\right)$ and weight average $\left(M_{w}\right)$ molecular weights, polydispersity $(D)$, random versus block copolymers, composition of blends, branching, and tacticity. Accurate determination of some of these values requires that molecular ions of each oligomer species be produced during ionization without fragmentation and that the ionization, instrument transmission, and detection efficiencies be known as a function of mass and preferably not altered as a function of stereochemistry or reasonable changes in chemical composition.

The MALDI technique has been shown to produce ions from several types of synthetic polymers. The accuracy of the molecular weight distributions obtained by this technique previously have been assessed by comparison to other polymer characterization methods such as SEC, light scattering, and so forth [17-21]. Montaudo et al. [19] observed that $M_{\mathrm{p}}$ (most probable peak) values were consistently low for methylmethacrylate (MMA) polymers as measured by MALDI in comparison to SEC $M_{\mathrm{p}}$ values. Burger et al. [18] used partially isolated oligomers of poly $(R)$-3-hydroxybutanoate to determine that MALDI discriminates against low molecular weight (1540-u) oligomers for these polymers, but that for molecular weights between 2900 and 8400 the response is molar. They were able to calculate an ionization response factor for the low mass discrimination. Lehrle and Sarson [20] observed that MALDI gives low molecular weight values relative to SEC for a narrow polydispersity poly(methylmethacrylate) (PMMA) standard. These 
authors suggested that laser-induced fragmentation or preferential desorption of low mass oligomers could account for the low MALDI values.

Here we report on the utility of MALDI as a means to characterize low molecular weight methacrylate polymers. Isolated syndiotactic and isotactic poly(methylmethacrylate) oligomers that contain 25 and 50 repeat units were used to assess propensity for MMA oligomers to fragment under MALDI conditions and to determine if observed ion currents are independent of oligomer mass. We previously reported that if peak area compression is taken into account in peak area calculation, the response by ESI mass spectrometry for these PMMA oligomers is molar and fragmentation is not observed [15]. It is not apparent that a molar response will be obtained for oligomers in MALDI because a molar response usually is not observed for peptide or protein mixtures [22].

\section{Experimental}

Mass spectra were obtained on the following time-offlight (TOF) mass spectrometers: a Fisons TOFSpec (Manchester, England), a Finnigan VISION-MAT 2000 (Bremen, Germany), and a Bruker Reflex (Bremen, Germany). Data were obtained in both the linear and reflectron modes on the Vision 2000 and the Bruker Reflex instruments. The deflector ion impact energy (the combined source acceleration voltage and detector postaccelerating voltage) was either 25 or $30 \mathrm{kV}$, unless otherwise noted. The Vision 2000 was used for those experiments in which $6-\mathrm{kV}$ ion impact voltage was indicated by operating in the reflectron mode with $1-\mathrm{kV}$ postacceleration. Nitrogen lasers $(337 \mathrm{~nm})$ were used with beam attenuation set to be near ion appearance threshold.

PMMA samples were obtained from Polymer Laboratories (Church Stretton, UK). The individual MMA oligomers were supplied by Professor K. Hatada (Osaka University, Japan). The samples were isolated from isotactic and syndiotactic MMA oligomers by using preparative supercritical fluid chromatography $[23,24]$. The amounts of each oligomer collected ranged from 0.1 to $0.7 \mathrm{mg}$. Equimolar solutions were obtained from weighed quantities of each isolated oligomer. Matrix materials, trans-3-indole acrylic acid (IAA; ICN Biochemicals, Cleveland, $\mathrm{OH}$ ), 2,5-dihydroxybenzoic acid (DHB; Sigma, St. Louis, MO), and [2-(4-hydroxyphenylazo)-benzoic acid] (HABA; Aldrich, Milwaukee, WI) were used as received from the manufacturer without purification. The PMMA samples were run by using IAA. The individual MMA oligomers were run in IAA, DHB, and HABA.

Samples were prepared by dissolving the PMMA or MMA oligomer samples in either chloroform or acetone to make a solution concentration of $\sim 20 \mathrm{pm} / \mu \mathrm{L}$. Two microliters of the polymer solutions were mixed with $8 \mu \mathrm{L}$ of the matrix solution. The matrix solution was prepared in either chloroform or acetone at a concentration of 5-mg matrix per milliliter of solvent. The sample and matrix were mixed and $\sim 0.5 \mu \mathrm{L}$ were spotted under ambient conditions on the stainless steel target.

\section{Results and Discussion}

Figure 1 is the MALDI mass spectrum of a molecular weight standard PMMA with $M_{\mathrm{p}}=5270$ (SEC). Table 1 shows the comparison of $M_{\mathrm{p}}, M_{\mathrm{n}}, M_{\mathrm{w}}$, and $D$ values measured by MALDI and those supplied by the manufacturer (SEC). With the exception of polydispersity ( $D)$, all molecular weight values measured by MALDI are low in comparison to the SEC values. PMMA standards (MW $<50,000$ ) have been shown to result in MALDI spectra that are lower in mass than expected in comparison to the manufacturers' values of $M_{p}$ [19]. Even after correction for the expected differences in the $M_{\mathrm{p}}$ values measured by SEC and MALDI, the MALDI values are lower than expected. The number assigned to the most probable peak $\left(M_{\mathrm{p}}\right)$ in a polymer distribution is dependent on how the data are plotted [25]. Low $M_{w}$ values by mass spectrometry may be the result of fragmentation, or mass discrimination in the ionization, transmission, or detection of ions, or possibly a matrix effect.

\section{Fragmentation of Methylmethacrylate Oligomers}

MALDI mass spectra of peptides and small proteins generally show no significant fragment ions under MALDI-TOF conditions. Therefore, fragmentation of MMA oligomers was not expected and is not observed in the MALDI-TOF spectra of polymer distributions. However, fragmentation in which monomer (repeat) units are lost would not be observed in a spectrum of a distribution of oligomers because the fragment ions and molecular ions would overlap.<smiles>COC(=O)C(C)CC(C)(CC(C)(CC(C)C(=O)OC)C(=O)OC)C(=O)OC</smiles><smiles>CCC(C)CC(C)(CC(C)(C(=O)OC)C(=O)OC)C(=O)OC</smiles> 


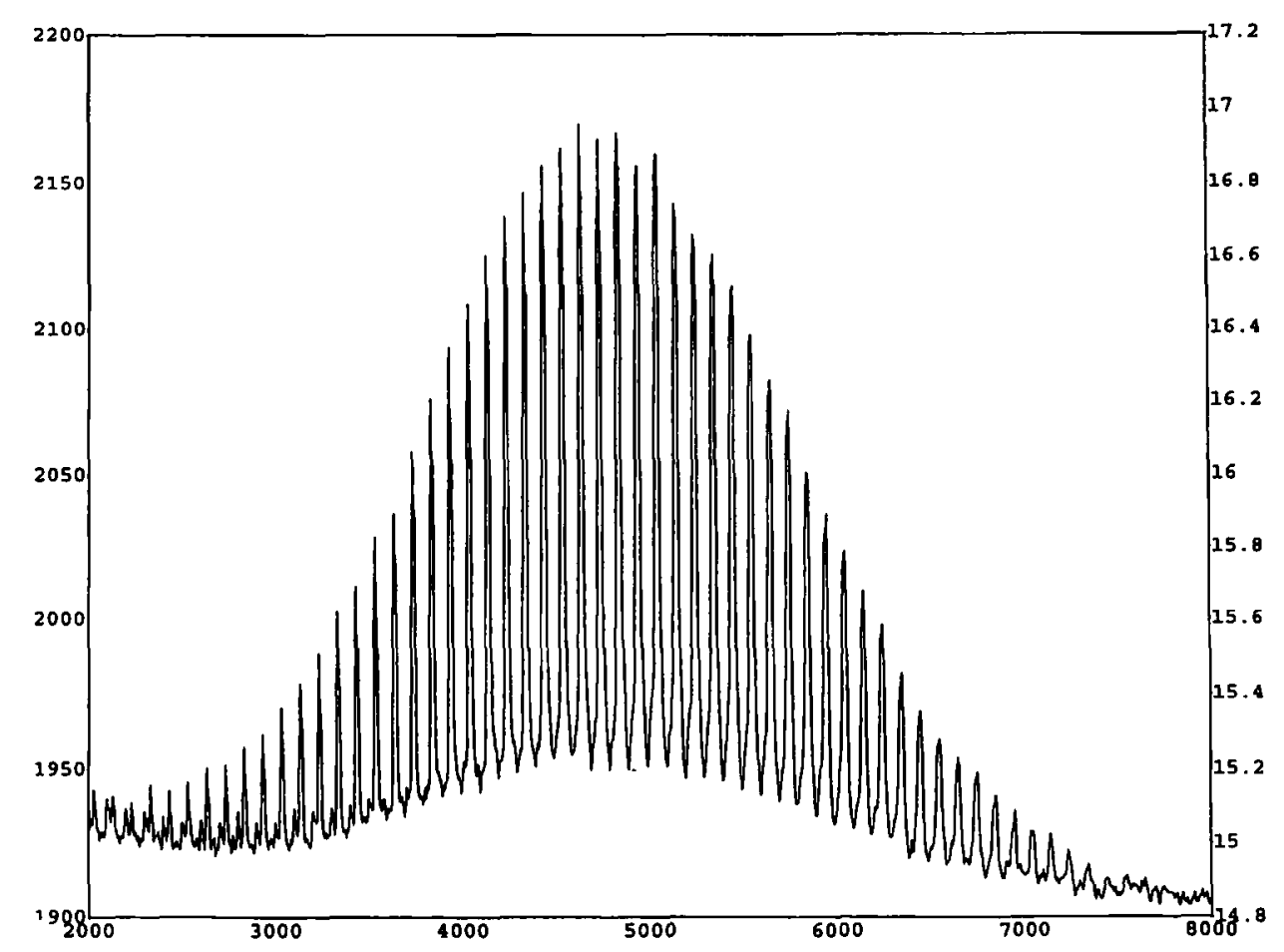

Figure 1. MALDI-TOF mass spectrum of PMMA 5270 that used indole acrylic acid (IAA) as the matrix and laser irradiation at $337 \mathrm{~nm}$ acquired in the reflectron mode.

Table 1. A comparison of MALDI and SEC molecular weight values for PMMA 5270

\begin{tabular}{ccc}
\hline & MALDI & SEC \\
\hline \hline$M_{\mathrm{p}}$ & 4600 & 5270 \\
$M_{\mathrm{n}}$ & 4625 & 4977 \\
$M_{\mathrm{w}}$ & 4890 & 5256 \\
$D$ & 1.06 & 1.06 \\
\hline
\end{tabular}

MALDI mass spectra of isolated isotactic and syndiotactic MMA oligomers with 25 and 50 repeat units were obtained with three different matrix materials. As can be seen for the MALDI reflectron mass spectrum of a mixture of the 25-mer and 50-mer (Figure 2) and the linear mass spectrum (Figure 3 ), only the molecular ions are observed. At mass-to-charge ratio values lower than those shown in the figures, matrix ions are observed. The abundance of the matrix ions varies according to the sample preparation.

Fragmentation is not observed in MALDI-TOF in either the linear or reflectron modes of operation for MMA oligomers to at least MW 5000. Fragmentation that will not be observed can occur in either the linear or normal reflectron modes of operation, but these fragment ions will not alter the MW distribution unless the production of fragment ions is mass dependent. Fragmentation was not observed with IAA, DHB, or HABA matrices, nor does a moderate increase in laser power cause fragmentation. Therefore, fragmentation in the mass spectrum of MMA does not cause the low values measured for $M_{\mathrm{p}}$ by using MALDI-TOF.
Ion Current Detection as a Function of Oligomer Mass

Weighed quantities of the isolated isotactic and syndiotactic MMA 25-mers and 50-mers each were dissolved in chloroform to make known molar solutions. Equimolar $( \pm 5 \%)$ chloroform solutions of the 25-mers and 50-mers were prepared and premixed with three different matrix solutions. MALDI mass spectra of these solutions were obtained on three different MALDI-TOF instruments to determine the molar response to each oligomer. These measurements are a combined response for the efficiencies of ion production, transmission, and detection, but, in addition, reflect the matrix used, sample-matrix preparation, laser wavelength and energy, and sampling of the target in the summed scans.

Figure 2 is the MALDI mass spectrum for an equimolar mixture of isotactic (it) 25 -mer and 50 -mer obtained by using a reflectron TOF instrument. The equimolar syndiotactic (st) mixture obtained in the linear mode is shown in Figure 3. As previously observed for ESI [15], the peak area for the 50-mer is equal to the peak area observed for the 25-mer within the reproducibility of the experiments. This was found when the detector ion impact voltage (combined acceleration and postacceleration voltages) was 25 or $30 \mathrm{kV}$. The ratio was not changed by a $5 \times$ dilution of the mixture. The equimolar response is observed even though detection of the 25-mer is predominately by ejection of secondary electrons from the first (conver- 


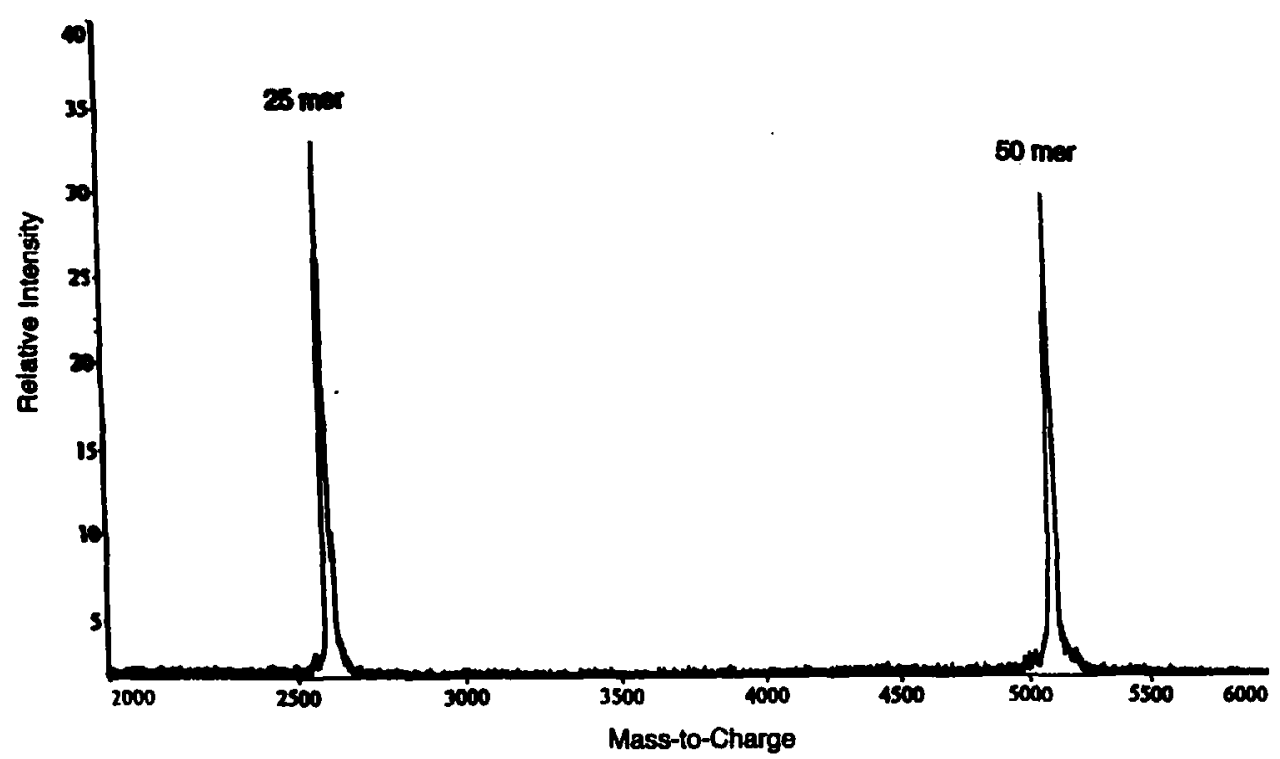

Figure 2. MALDI-TOF mass spectrum of an equimolar isotactic mixture of MMA oligomers with 25 and 50 repeat units that used DHB as the matrix acquired in the reflectron mode.

sion) dynode (i.e., ion to electron conversion), whereas detection of the 50 -mer is more influenced by ejection of secondary ions from the conversion dynode. (Note the peak doublets in Figure 2: low mass is electron ejection and high mass is ion ejection. The ion doublet is observed more clearly in Figure 4, but at $6-\mathrm{kV}$ ion impact, secondary ion detection is suppressed.) In addition, an equimolar response was obtained for the isolated oligomers by using HABA, IAA, and DHB as matrix materials and on three different MALDI-TOF instruments.

The results were quite different when the postacceleration voltage was reduced from 20 (Figure 2) to $1 \mathrm{kV}$ (Figure 4) to give an ion impact voltage of only $6 \mathrm{kV}$. Figure 4 shows that under these conditions the 50-mer was approximately $40 \%$ the abundance of the 25 -mer. Thus, MALDI does discriminate, as expected, against high mass ions at lower detector impact energies. However, within the mass range of these experiments, $25-\mathrm{kV}$ ion impact is sufficient to eliminate detector mass discrimination. These results suggest the capability of MALDI to provide accurate $M_{\mathrm{p}}$ values for PMMA oligomer distributions in the mass range of these experiments.

In these experiments, the oligomers changed only by the number of identical monomer units. This work did not determine the effect that changing, for example, the end groups has on ionization. Peptides can have large chemical differences and do not generally give molar ion currents by MALDI [22]. A similar

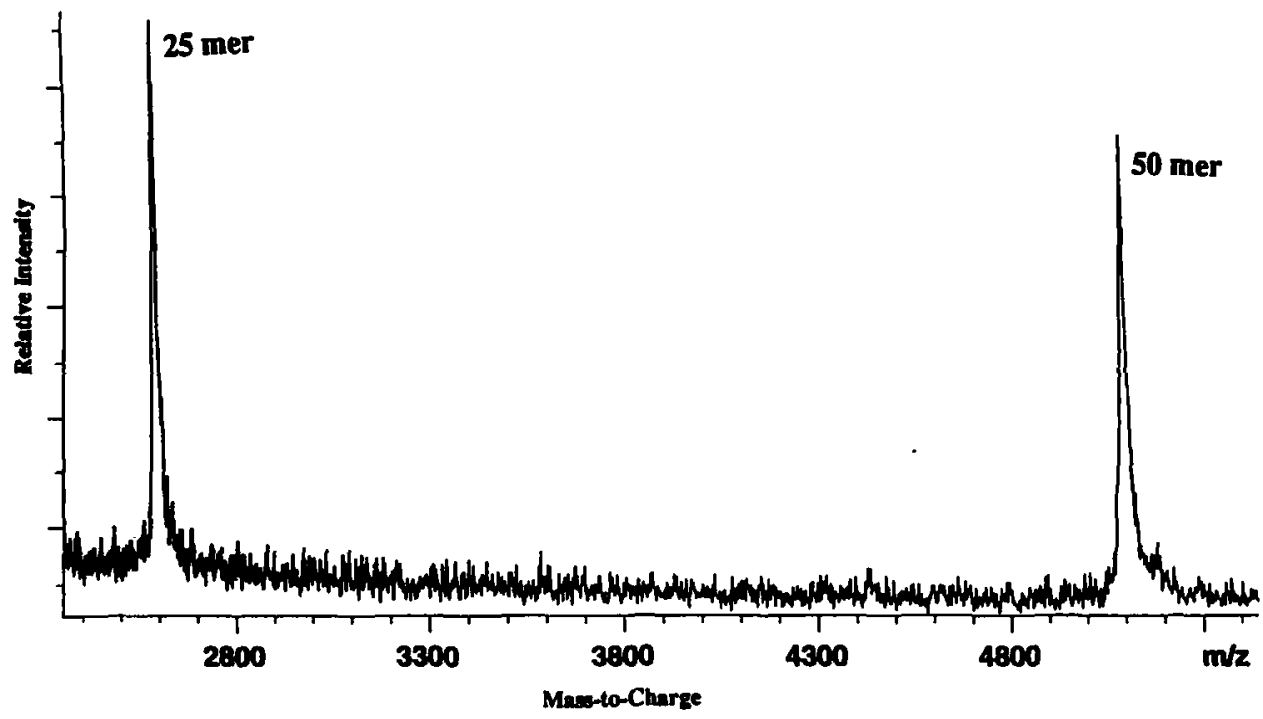

Figure 3. Linear MALDI mass spectrum of an equimolar mixture of syndiotactic MMA 25 mer and 50 mer that used DHB as matrix. 


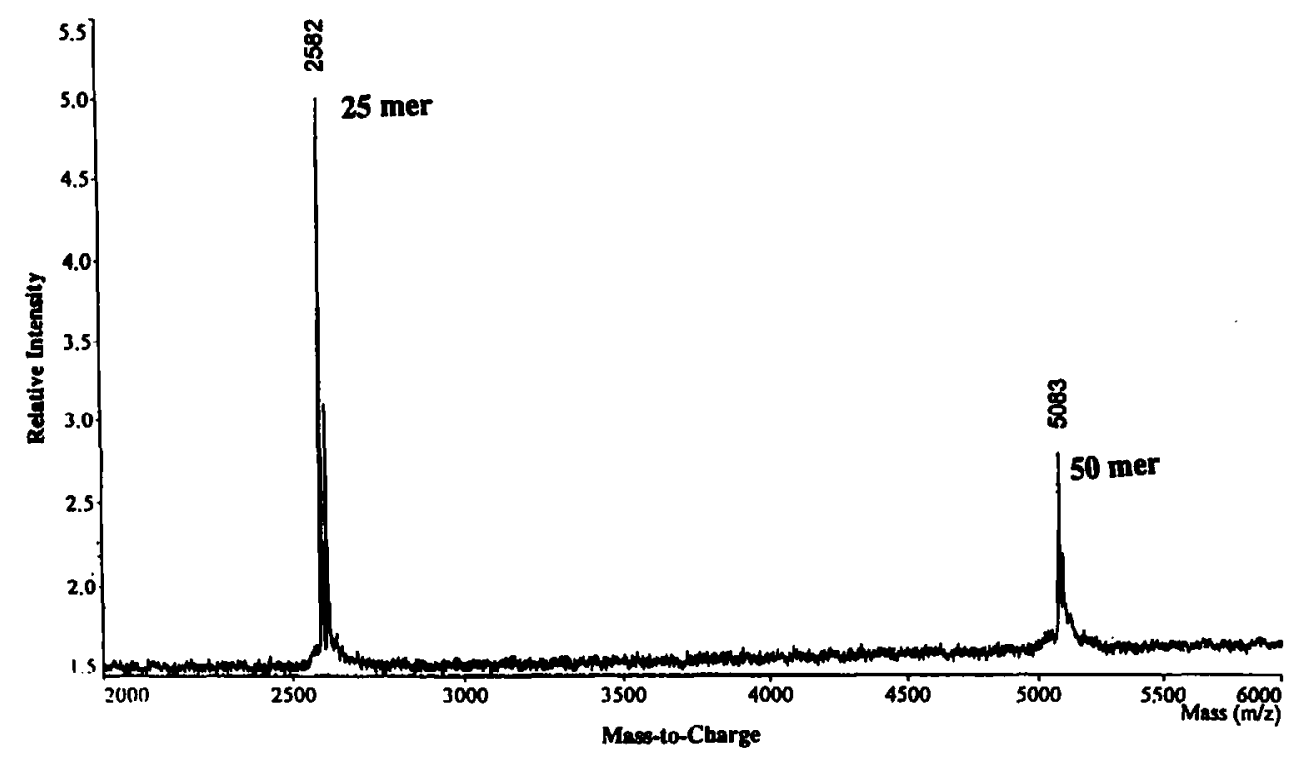

Figure 4. MALDI mass spectrum of an equimolar mixture of isotactic MMA 25-mer and 50-mer with a detector ion impact of $6 \mathrm{kV}$ (DHB matrix).

effect is expected for polymer molecules with large chemical differences.

\section{Determination of Stereochemistry in Methylmethacrylate Oligomers}

MALDI mass spectra were obtained for each of the MMA stereoisomers. Only molecular ions were observed in either the linear or reflectron modes for all isomers. Mixture of the it-25-mer with an equimolar amount of st-50-mer gave, within experimental error, equal signals at ion impact energies of $25 \mathrm{kV}$ or higher. Any effects of stereochemistry on the production of ions for these samples were too subtle to detect in these experiments.

\section{Conclusion}

MALDI-TOF is shown to give a molar response for MMA oligomers that have molecular weights between 2500 and $5000 \mathrm{u}$ provided that the detector impact energy is sufficiently high (in these experiments 25 or $30 \mathrm{kV}$ ). Furthermore, no fragmentation of these oligomers was observed in either the linear or reflectron modes of operation. Ionization efficiency was observed not to be a function of tacticity of the oligomers. These data suggest that at least for narrow PMMA oligomer distributions, MALDI can produce a distribution that is representative of the number of each major oligomer species present in the mixture. Thus, MALDI should give an accurate number average determination of $M_{\mathrm{p}}$ in a PMMA distribution because this determination is made over a narrow mass range by using the most intense peaks. This work did not assess ionization as a function of chemical changes such as oxidation, end group differences, and so forth.

\section{Acknowledgments}

We thank Professor Koichi Hatada and Dr. Koichi Ute from the Faculty of Engineering Science at Osaka University for providing the isolated oligomers. We graciously acknowledge John Race and Tom Driscoll of Fisons for the loan of a Fisons VG TOFSpec mass spectrometer and Dr. R. Schubert of Finnigan and Dr. F. Mayer of Bruker for providing experimental data used in this paper.

\section{References}

1. Vouros, P.; and Wronka, J. W. In Modern Methods of Polymer Characterization, Barth H. G.; Mays, J. W., Eds.; Wiley: New York, 1991; Chap. 12.

2. Cook, K. D., In Encyclopedin of Polymer Science and Engineering, 2nd ed.; Wiley: New York, 1987; Vol. 9, p 319.

3. Sulten H-R.; Lattimer, R. P. Mass Spectrom. Rev., 1994, 3, 231.

4. Rollins, K.; Scrivens, J. H.; Tylor, M. J.; Major, H. Rapid Commun. Mass Spectrom. 1990, 4, 355.

5. Prokai, L. Field Desorption Mass Spectrometry; Marcel Dekker: New York, 1990; Vol. 9.

6. Cochran, R. L. Appl. Spectrosc. Rev. 1986, 22, 137.

7. van Leysen, D.; Hagenhoff, B.; Niehuis, E.; Benninghoven, A.; Bletsos, I. V.; Hercules, D. M., J. Vac. Sci. Technol., A 1989), 7, 1790.

8. Hercules, D. Polym. Mat. Sci. 1988, 59, 724.

9. Tanaka, K.; Waki, H.; Ido, Y.; Akita, S.; Yoshida, Y.; Yoshida, T. Rapid Commun. Mass Spectrom. 1988, 8, 151.

10. Tanaka, K.; Waki, H.; Ido, Y.; Akita, S.; Yoshida, Y.; Yoshida, T. Rapid Commun. Mass Spectrom. 1988, 8, 151.

11. Nuwaysir, L. M.; Wilkins, C. L.; Simonsick, W. J., Jr. J. Am. Soc. Mass Spectrom. 1990, 1, 66.

12. Simonsick, W. J. Jr.; Hatada, K.; Xi, F.; Vogl, O. Macromolecules 1990, 23, 4470.

13. Bombick, D.; Pinkston, J. D.; Allison, J. Anal. Chem., 1984, 56, 396.

14. Fenn, J. B.; Mann, M.; Meng, C. K.; Wong, S. F.; Whitehouse, C. M. Mass Spectrom. Rev. 1990, 9, 37. 
15. McEwen, C. N.; Simonsick, W. J.; Larsen, B. S.; Ute, K.; Hatada, K. J. Am Soc. Mass Spectrom. 1995, 6, 908.

16. Danis, P. O.; Karr, D. E.; Mayer, F.; Holle, A.; Watson, C. H. Org. Mass Spectrom. 1992, 27, 843; Danis, P. O.; Karr, D. E. Org. Mass Spectrom., 1993, 28, 923.

17. Bahr, U.; Deppe, A.; Karas, M.; Hillenkamp, F.; Giessman, U. Anal. Chem. 1992, 64, 2866.

18. Burger, H. M.; Muller, H. M.; Seebach, D.; Burnsen, K. O.; Schar, M.; Widmer, H. M. Macromolecules 1993, 26, 4783.

19. Montaudo, G.; Montaudo, M. S.; Poglisi, C.; Samperi, F. Rapid Commun. Mass Spectrom., 1995, 9, 453.
20. Lehrle, R. S.; Sarson, D. S. Rapid Commun. Mass Spectrom., $1995,9,91$.

21. Danis, P. O.; Karr, D. E.; Simonsick, W. J. Jr.; Wu, D. T. Macromolecules, 1995, 28, 1229.

22. Olumee, Z.; Sadeghi, M.; Tang, X.; Vertes, A. Rapid Commun. Mass Spectrom. 1995, 9, 744.

23. Ute, K.; Miyatake, N.; Asada, T.; Hatada, K. Polym. Bull. 1992, 28, 561.

24. Ute, K.; Miyatake, N.; Osugi, Y.; Hatada, K. Polym. J. 1993, $25,1153$.

25. Jackson, C.; Larsen, B.; McEwen, C. Anal. Chem. 1996, in press. 